\title{
A la Recherche du héros dans \\ Les Liaisons dangereuses
}

George Nahrebecky

(St. Mary's University, Halifax, Nova Scotia)

La notion de héros littéraire résiste depuis longtemps à toutes les tentatives d'en faire une analyse exhaustive. Cela n'est guère surprenant, étant donné que le terme "héros" lui-même, doué d'une multiplicité de sens, chevauchant les domaines social, idéologique, culturel et littéraire, entre autres, ne se laisse pas facilement saisir. En effet, ce que les sociétés ont investi d'une connotation de supériorité (le héros est celui qui se distingue des autres par des qualités dont la plupart des membres d'une société donnée ne jouissent pas) a souvent été rabaissé dans son acception littéraire, d'où la classification du héros littéraire de Northrop Frye, ${ }^{1}$ ou bien le concept d' "anti-héros". Nombreuses sont les théories qui prétendent pouvoir isoler le héros des autres personnages d'un texte à partir de certains critères. Chez Propp, c'est celui à qui est confiée une quête quelconque. Selon Tomachevski, le héros se définit comme "le personnage suivi par le lecteur avec la plus grande attention....celui qui reçoit la teinte émotionnelle la plus vive et la plus marquée"2, hypothèse qui débouche sur une problématique relevant du domaine de la réception. En effet, la notion de "teinte émotionnelle" est à manipuler avec soin, car elle dépend évidemment autant du lecteur que du producteur du texte. Non moins intéressante et tout aussi problématique est l'hypothèse de Philippe Hamon, selon laquelle le héros pourrait être "le personnage au portrait le plus riche, à l'action la plus déterminante, à l'apparition la plus fréquente." 3

Il est à noter que les deux définitions proposées jusqu'ici relèvent de deux niveaux textuels bien différents. Celle de Tomachevski, qui tient aux rapports d'ordre "émotionnel" implique nécessairement une certaine subjectivité de la part du lecteur qui, à son tour, entraîne des considérations d'ordre idéologique, normatif, moral, etc. En revanche, l'hypothèse de Hamon, ainsi que celle de Propp, sont essentiellement d'ordre textuel, et par conséquent, sujettes à une vérification beaucoup plus rigoureuse.

LittéRéalité, Vol. I, No. 1, Printemps/Spring 1989 
Dans le cadre de la problématique du héros nous nous proposons une étude des Liaisons dangereuses de Laclos. Il s'agit d'un texte qui, dans la mesure ou il met en scène trois personnages qui peuvent chacun prétendre au titre de "héros" ou bien d' "héróine" selon une ou plusieurs acceptions du mot, semble lancer un défi à l'idée d'une typologie rigoureuse du héros de roman.

Une analyse des Liaisons dangereuses dans la perspective du héros romanesque risque de soulever certains problèmes fondamentaux qui ont trait non seulement au récit lui-même, mais à sa narration. Là où l'un des actants d'un roman est également impliqué dans sa narration il y a lieu de s'interroger sur les rapports entre le statut d'un tel actant dans une perspective sociale et son statut dans une perspective narratologique.

Le narrateur intradiégétique, focalisateur d'un récit donné aurait d'emblée avantages sur le plan héroïque. Car celui qui parle de lui-même, de ses propres exploits, est le héros de son propre discours. Toutefois, dans le cas d'un roman par lettres. le récit, ainsi que sa narration, et par extension sa focalisation, sont fragmentés, entrelacés dans un réseau complexe. Dans le cas

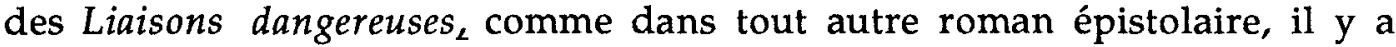
syncrétisme entre le destinateur d'une lettre donnée et le narrateur du roman, vu que chaque lettre constitue en elle-même une instance narrative. Il y a lieu alors, dans un premier temps, de procéder à une évaluation quantitative de la production de lettres de chaque actant.

Aussi faut-il sans doute accorder quelque importance non seulement au destinateur d'une lettre donnée, mais également à son destinataire, bien que l'importance de celui-ci soit évidemment moindre. Comme Gerald Prince le souligne dans son étude du narrataire: "(...) si le protagoniste ou la personnalité marquante d'une narration y joue souvent le rôle de narrateur et s'affirme en tant que tel (...) il n'y a pas (...) de héros qui soit avant tout narrataire."4 Pourtant, s'il s'agit d'attirer l'attention du lecteur, il faut admettre que le destinataire d'une lettre y est pour quelque chose, ne serait-ce que par le fait que celui-ci est nommé de façon explicite. "Le Vicomte de Valmont à la Marquise de Merteuil" (c'est nous qui soulignons) signifie qu'à la surface, c'est à la Marquise, et à elle seule qu'est destiné le discours de Valmont. Il s'agit d'un drame en quelque sorte extra-diégétique qui se déroule entre deux actants. Si le récit est en effet focalisé par celui qui narre, (dans ce cas-ci Valmont), c'est le narrataire qui constitue, pour ainsi dire, l'écran sur lequel le discours focalisé est projeté. Chaque instance narrative, c'est-à-dire chaque lettre, constitue en elle-même, une espèce de "microdrame" à l'intérieur du drame englobant qu'est le récit pris en sa totalité. Par 
conséquent, le fait d'être narrataire à un moment donné revêt lui aussi une certaine importance (ce qui, comme nous allons le voir, est surtout évident dans le cas de Mme de Merteuil).

En évaluant l'importance de chaque personnage uniquement en fonction de sa correspondance épistolaire, c'est-à-dire, de sa fonction en tant qu'émetteur/récepteur de lettres, c'est le Vicomte de Valmont qui joue un rôle prépondérant. Il est l'auteur de 51 lettres, soit presque du tiers de la production totale du texte, qui est de 175 lettres. De plus, il en reçoit 37, ce qui veut dire qu'il est impliqué directement, en tant que destinateur/destinataire dans 50 pour cent des lettres échangées. C'est également Valmont qui correspond avec le plus grand nombre de personnages différents. Il entretient une correspondance suivie avec Mme de Merteuil, ainsi qu'avec la présidente de Tourvel, et il écrit à Cécile, à Danceny, au père Anselme et à Azolan. Pour ce qui est des rapports épistolaires directs, Valmont l'emporte sur la Marquise de Merteuil, qui est l'auteur de 28 lettres, (16 pour cent de la corréspondance totale) dont 22 qui sont adressées à Valmont, deux à Cécile, deux à Mme de Volanges et deux à Danceny. La Marquise en reçoit 41, ce qui veut dire qu'elle est destinatrice/destinataire de 69 lettres, environ 39 pour cent de la production totale. Il y a 24 lettres signées par la présidente de Tourvel (14\%) qui en reçoit 19 , soit un total de 43 lettres, ou 25 pour cent de la production totale.

C'est alors Valmont qui se trouve en tête du paradigme des émetteurs au niveau épistolaire, et qui, par conséquent, domine la structure narrative du roman.

$\begin{array}{lrrrr}\text { émetteur } & \text { lettres } & \text { récepteur } & \text { lettres } & \text { total } \\ \text { Valmont } & 29 \% & \text { Valmont } & 21 \% & 50 \% \\ \text { Merteuil } & 16 \% & \text { Merteuil } & 23 \% & 39 \% \\ \text { Tourvel } & 14 \% & \text { Tourvel } & 11 \% & 25 \%\end{array}$

Tout cela, on le sait bien, est peu concluant: Le fait que Valmont écrit le plus grand nombre de lettres lui permet de s'imposer en tant que narrateur principal, ce qui a pour effet d'accentuer son importance sur le plan de la narration de l'histoire sans pour autant l'établir comme personnage principal au niveau de la structure interne. Il est évident toutefois, que dans le cas de narrateurs intra-diégétiques multiples où, comme le prétend Genette, "la focalisation sur le narrateur est en même temps focalisation sur le héros", 5 il 
faut tenir compte du dossier de chaque actant à partir d'une perspective narratologique. En revanche, il est tout aussi bien évident que l'on ne peut pas s'attendre à ce que la narratologie puisse jeter trop de lumière sur ce qui relève aussi d'autres niveaux d'analyse.

En effet, il y a lieu de s'interroger sur le statut de chaque actant à partir des procédés mis en oeuvre pour attirer l'attention du lecteur. Or, à titre heuristique, on peut réduire le nombre de critères permettant une évaluation de la "performance" de chaque actant à cet égard à ceux de Hamon cités cidessus, à savoir: l'étendue du programme descriptif de tel personnage, l'importance et la portée de ses actions vis-à-vis du déroulement de l'intrigue, et le nombre, la durée et l'importance relative de ses interventions.

En ce qui concerne les programmes descriptifs des actants des Liaisons, il faut souligner qu'il y a une pénurie de données portant sur l'aspect physique de tous les personnages principaux. Dans le cas de Valmont, à part sa "belle figure, pur effet du hasard" (lettre 81), on ignore tout, jusqu'à son âge. Chez Merteuil, à part quelques allusions qui connotent une certaine beauté (...nos plus jolies femmes... sont encore si loin de vous... [lettre 18]), le physique fait aussi défaut. Mme de Tourvel, elle, se voit l'objet d'une appréciation double, celle de Merteuil, selon laquelle elle serait "passablement faite, sans grâces" (5) et celle, un peu plus flatteuse, de Valmont, qui lui attribue "les plus belles dents du monde" et une "figure céleste" (6).

Quoi qu'il en soit, le manque de détails physiques supplémentaires, ou bien l'existence d'une certaine homogénéité sur le plan physique (tous les actants sont plus ou moins bien faits) empêche le genre d'analyse binaire qui se prête à certains types littéraires, et selon laquelle la beauté s'identifie au bien et par extension, à la laideur au mal et par extension, à la scéleratésse. ${ }^{6}$ Il suffit de penser aux contes folkloriques où en principe les héros sont beaux et leurs opposants marqués de traits de laideur. En effet, quel que soit le genre considéré, l'actant-sujet est presque toujours mieux fait que son opposant. Ainsi, même un héros "naturaliste" comme Etienne Lantier est "supérieur" sur le physique à son opposant Chaval.

Dans les Liaisons, pourtant, il y une transformation sur le plan de l'opposition beauté -/- laideur. Il s'agit d'une transformation physique et esthétique, à savoir, le défigurement de Mme de Merteuil, qui est d'autant plus remarquable qu'il correspond à une modification sur le plan actanciel. Or, si on tient compte du fait qu'au début du roman c'est Mme de Merteuil qui confie à Valmont la mission de séduire Cécile on peut prétendre que c'est à 
Mme de Merteuil qu'il faut attribuer la fonction de destinatrice alors que Valmont, chargé, en devient actant-sujet. Toutefois, vers la fin du roman, après la déclaration de guerre entre Valmont et Mme de Merteuil, celle-ci devient manifestement son opposante. Il y a donc transformation de fonctions dan le cas de cette dernière, qui quitte son rôle de destinatrice, pour assumer le rôle de l'adversaire de Valmont. Le passage de Merteuil d'un rôle à l'autre est suivi d'une transformation analogue sur le plan de l'opposition beauté -/- laideur. Mme de Merteuil, peu après avoir causé la mort et par conséquent triomphé de Valmont, sera "affreusement défigurée". Victime de la petite vérole, elle s'en trouvera borgne et "vraiment hideuse" (175). Selon la convention qui relie la notion de héros à la beauté physique on peut même en déduire qu'elle est ainsi disqualifiée comme héroïne.

La métamorphose de Mme de Merteuil mise à part, les trois personnages principaux se trouvent plus ou moins sur un pied d'égalité quant à leur caractérisation au niveau physique, ce qui indique sans doute que tout genre de recensement de traits physiques en vue d'une hiérarchisation éventuelle est déconseillé. Pourtant, on peut constater certains écarts qui permettent une hiérarchisation selon l'importance relative des portraits psychologiques de nos héros éventuels. On constate alors que c'est encore Valmont qui s'impose à cet égard mais non pas, comme on pourrait s'y attendre, en vertu de sa domination épistolaire. Ce qui fait qu'il jouit d'une caractérisation psychologique dominante, c'est d'une part que dans ces lettres il a tendance à parler de lui-même et d'autre part, qu'il fait parler de lui-même. Dans la plupart des lettres de Mme de Tourvel à Mme de Volanges et à Mme de Rosemonde, il est justement question de Valmont. Mais ce qui est encore plus important, c'est que dans beaucoup de ces lettres à Mme de Merteuil, il se confesse, révélant ainsi certains traits de caractère qui, évalués selon les normes qu'il établit lui-même, doivent être perçus comme des faiblesses. Psychologiquement, il se met à nu devant elle à plusieurs reprises et cela tout au long du roman. Il y a donc un effet double: d'une part, Valmont est rehaussé quant à son statut de héros selon les critères que nous avons établis, c'est-à-dire, en attirant l'attention du lecteur sur lui-même; d'autre part, il est rabaissé car, en jugeant d'après la doctrine libertine qu'ils prônent tous les deux, il est plus faible que Mme de Merteuil. Nours reviendrons sur ce point précis plus tard.

Après Valmont, c'est Tourvel qui manifeste le désir le plus aigu de s'épancher. Tourmentée par sa passion naissante et se trouvant dans l'impossibilité de l'intégrer dans son éthique, elle incarne l'univers tragique. Mais dans Les Liaisons dangereuses, c'est un univers qu'elle partage par voie 
épistolaire avec Mme de Rosemonde. Il en résulte un profil psychologique assez détaillé de Mme de Tourvel. Quant à Mme de Merteuil, à part la lettre 81 (où elle raconte sa vie à Valmont de façon assez détaillée), elle a tendance à renoncer au genre d'épanchement émotionnel pratiqué par Valmont et Tourvel. Par conséquent, bien que le lecteur connaisse bien sa nature - on sait qu'elle est rusée, cynique, méprisante et pas à l'abri d'une certaine méchanceté - son portrait psychologique (et physique d'ailleurs) est légèrement plus faible que celui de Valmont et de Mme de Tourvel. C'est en effet ce que Todorov reproche à l'intégrité de la structure des Liaisons.

[La] faible intégration de Mme de Merteuil dans le réseau des personnages est d'ailleurs un des rares défauts de la composition du roman; ainsi le lecteur n'a pas suffisamment d'indication sur son charme féminin qui joue pourtant un si grand rôle dans le dénouement. 7

C'est un point de vue que nous ne partageons pas, car, comme nous espérons pouvoir le montrer plus tard, ce que Todorov qualifie de "faible intégration" et que nous qualifierions plutôt de "distanciation actanctielle" permet à Merteuil d'accéder au sommet d'une autre hiérarchie. Il est à noter toutefois, que le statut de Mme de Merteuil sur le plan épistolaire, où elle est en deuxième position, ne correspond pas à son statut sur le plan des programmes descriptifs, où elle est en bas de la liste.

En ce qui concerne le dernier critère, c'est-à-dire l'importance de chaque actant par rapport au déroulement de l'intrigue, il n'est pas difficile de constater que c'est Valmont qui en constitue en quelque sorte l'armature. Il entretient des rapports avec tous les personnages importants du roman. Il est impliqué dans les machinations de la Marquise de Merteuil, il corrompt la Présidente, séduit Cécile, et se bat en duel avec Danceny. C'est lui, plus que tout autre personnage $\mathrm{du}$ roman qui agit, non seulement sur le plan cérébral et épistolaire, mais également sur le plan physique. C'est lui qui est l'auteur de presque tout ce qui, dans le roman, relève du domaine de l'aventure, de l'action proprement dite, de son "acte de charité" à la campagne conçu dans le but de tromper la Présidente jusqu'à la séduction brutale de celle-ci vers la fin du roman. Ainsi, il satisfait à l'exigence que le héros soit le personnage "à l'apparition la plus fréquente". A ce niveau d'analyse, il n'y a vraiment pas d'autres actants qui lui font concurrence, bien qu'on puisse compléter la hiérarchie en constatant que, dans un sens physique, Mme de Merteuil est plus dynamique que Tourvel, dont le champ d'action se limite plutôt à des débats intérieurs. Il est évident donc que l'intrigue (et le récit lui-même), s'organisent autour de Valmont de façon centrifuge, à la manière des rayons 
d'une roue. Sans Valmont comme moyen, la structure du roman s'effondrait sur elle-même.

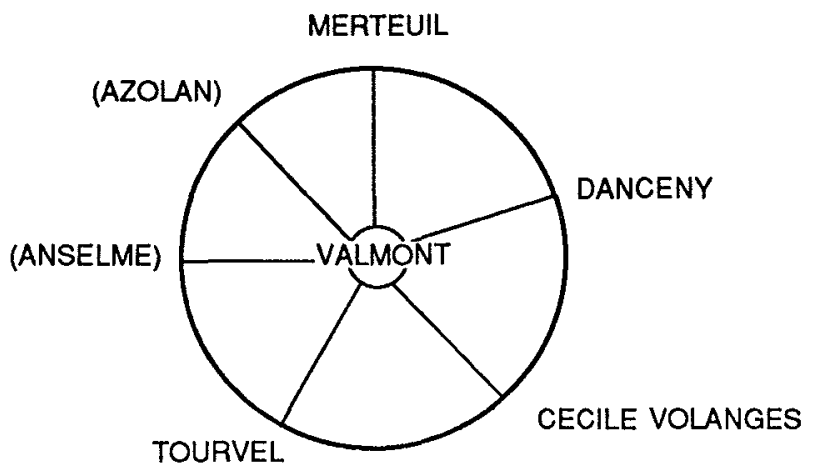

Notre schéma démontre assez clairement qu'afin d'agir les uns sur les autres, les personnages des Liaisons sont presque toujours obligés de passer par l'entremise de Valmont. Ce n'est que par son intervention auprès de Cécile que Merteuil espère pouvoir se venger de Gercourt. Danceny se fie à lui dans l'espoir de se faire aimer de Cécile. Il y a même des cas où il joue un rôle tout à fait passif, sans pour autant être moins indispensable en tant qu'élément permettant, ou bien justifiant, une communication épistolaire. Ainsi, Tourvel et Mme de Volanges s'écrivent avant tout pour parler de lui. Il s'en dégage une série de structures triadiques, toutes ayant comme élément constitutif le personnage de Valmont. Il y a les triades Merteuil - Valmont Tourvel, Merteuil - Valmont - Cécile, Danceny - Valmont - Cécile et Merteuil - Valmont - Danceny pour n'en nommer que quelques-unes.

D'autre part, notre schéma nous permet également de constater qu'on ne peut vraiment passe rendre compte de l'importance de Mme de Merteuil à partir de structures triadiques, et qu'il faut également parler, non seulement de forces centrifuges mais aussi de forces linéaires. S'il est vrai que Merteuil passe souvent par l'entremise de Valmont, il n'en est pas moins vrai qu'elle agit directement, sur Cécile, sur Danceny, et ce qui est plus important, sur Valmont. Or, si Valmont assure l'intégrité du circuit, le courant qui l'alimente provient de Merteuil. C'est en effet Merteuil qui constitue la force motrice de l'intrigue, force qui, en passant par les autres actants, et surtout par Valmont, se propage dans toutes les extrémités du réseau actoriel. C'est Mme de Merteuil alors qui dirige le déroulement de l'intrigue, qui incite les autres à agir selon ses desseins, presque à son gré. 


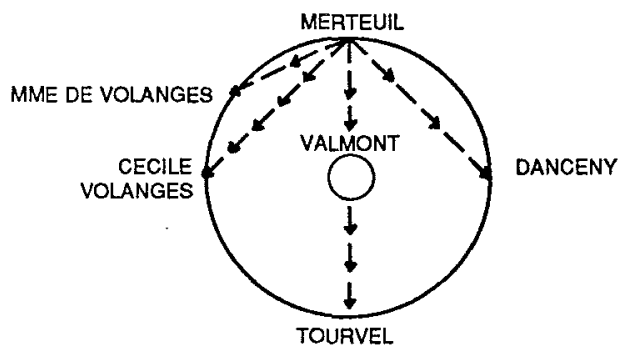

Voilà donc ce qui oppose Valmont et Merteuil sur le plan hérö̈que. Il s'agit en effet d'une relation de complémentarité, ou bien, aux fins de cette analyse, d'opposition entre le linéaire et le centrifuge. En outre, il nous semble que ce n'est qu'en opérant une procédure heuristique de soustraction que l'on peut se prononcer sur l'importance relative de chacun d'eux. Or on peut postuler qu'à partir d'une perspective purement structurale, l'histoire pourrait éventuellement se passer de Merteuil sans pour autant perdre son intégrité de base. Ainsi Valmont séduirait quand même Cécile et Mme de Tourvel et l'intrigue, avec quelques modifications, resterait intacte. Il n'en va pas de même pour Valmont. Sa disparition entraînerait inévitablement un échec, un court-circuit narratif et structural. A ce niveau d'analyse, même Mme de Tourvel s'avère aussi importante vis-à-vis de la structure du roman que Mme de Merteuil car, en supprimant le rôle de Mme de Tourvel, et par conséquent sa séduction par Valmont, on priverait le récit d'un des constituants dont il aurait beaucoup de mal à se passer. Sur le plan hiérarchique alors, par rapport à l'intégrité structurale du roman, Valmont constitue l'élément le plus important, suivi de Tourvel et de Merteuil.

En passant en revue les structures hiérarchiques qui se dégagent jusqu'ici, il semble bien que le récit soit dominé, à presque tous les points de vue, par Valmont et que c'est à lui qu'il faut décerner le titre de "héros" ou bien de "personnage principal", l'un étant, dans le cadre de cette étude l'équivalent de l'autre. En effet, c'est Valmont qui est en tête du paradigme des destinateurs de lettres, et qui domine la plupart des autres hiérarchies qui ont trait aux programmes descriptifs et à l'importance structurale de chaque actant des Liaisons.

Pourquoi donc les critiques s'obstinaient-ils à voir en Mme de Merteuil, et non pas en Valmont le personnage principal, (dans notre sens le héros) des Liaisons ${ }^{8}$ Il y a évidemment le fait, comme nous l'avons déjà remarqué, qu'elle se trouve en tête de ce que nous avons appelé la structure linéaire du roman, mais il y a sans doute plus que cela. S'il est vrai que Valmont et Mme de Merteuil sont tous deux intellectuellement supérieurs aux autres 
personnages, il n'en est pas moins vrai que Mme de Merteuil est supérieure à Valmont et qu'elle en est bien consciente: "Et qu'avez-vous donc fait, lui demande-t-elle, que je n'aie surpassé mille fois?" (81) Valmont lui-même se voit émerveillé par ses machinations diaboliques: "On ne peut que s'humilier devant la profondeur de vos vues, si on en juge par le succès de vos démarches" (64). De par son acuité intellectuelle extraordinaire, Merteuil est un personnage "surdoué", un être supérieur jusqu'à son statut social. (En tant que "Marquise", elle jouit d'un rang supérieur à celui du "Vicomte" et celui de la "Présidente"). C'est à elle, en sa qualité de femme supérieure, que les autres demandent conseil:

"La Marquise observe, mesure, réfléchit, étudie, médite élabore un savoir, mais ne s'en contente pas: ce savoir doit lui donner prise sur le monde et sur les autres, est promesse de jouissance et d'action". 9

Même Valmont a souvent l'air d'être motivé autant par le désir de susciter son approbation que par le besoin d'assouvir ses passions. Il n'est pas donc surprenant qu'elle soit en tête du pardigme des narrataires. Les pulsions narratives qu'elle déclenche viennent se répercuter sur elle, et c'est elle qui les relance, en les renouvelant, en leur donnant un nouvel essor. Elle devient donc un "être à part", une espèce de monstre héroïque. Ce que Todorov appelle "faible intégration" n'est autre chose qu'une distanciation par rapport aux autres personnages imposée par sa position de supériorité, distanciation qui, plutôt que d'être incongrue, est même nécessaire, car elle lui permet d'animer ou bien de présider, pour ainsi dire, le roman. S'il est évident que Valmont est en effet le héros des Liaisons dangereuses, et nous y croyons, il nous semble bien que c'est Mme de Merteuil qui le fait briller, et qui en a fait un chef-d'oeuvre.

Nous n'avons pas beaucoup parlé de la Présidente de Tourvel, bien que dans le domaine critique elle ne soit pas sans ses admirateurs. ${ }^{10}$ Mais il semble bien que tout en jouant un rôle structural important, indispensable même, elle ne puisse aucunement accéder au titre de héros qu'à partir de critères d'ordre moral, ce qui n'intéresse pas cette étude. Il n'y aurait sans doute pas d'idéologie occidentale qui ne rangerait pas Mme de Tourvel du côté du bien selon l'opposition bien - /- mal, ce qui aurait pour effet, comme nous l'avons déjà noté, de lui attribuer un certain statut héroïque qui ne serait pas sans rapport avec une connaissance de types littéraires. En effet, il se peut bien qu'elle soit, chez la plupart des lecteurs, le personnage "qui reçoit la teinte émotionnelle la plus vive et la plus marquée", ce qui ferait d'elle un héros en 
termes tomachevskiens. Pourtant, selon les structures hiérarchiques que nous avons établies, elle occupe, à une exception près, un rang inférieur et doit s'incliner, ne serait-ce qu'à l'égard de son statut héroïque ainsi défini, devant les "poids-lourds" des Liaisons dangereuses, c'est-à-dire, devant le Vicomte de Valmont et la Marquise de Merteuil.

Dans les Liaisons dangereuses, le problème du héros vu en tant que personnage principal dans une perspective structuraliste et même narratologique s'avère donc bel et bien réglable. Nous avons toutefois insisté sur la synonymie des termes "héros" et "personnage principal". Il suffit, on le sait bien, d'introduire la notion d'idéologie, entre autres, pour que notre notion de héros littéraire éclate, et que surgisse une problématique d'immense envergure, et d'une complexité étonnante. Idéologie du texte, idéologie hors texte, synchronique et diachronique, idéologie de la création/réception littéraires, la liste peut s'étendre de façon indéfinie. (Aussi faudrait-il sans doute rigoureusement définir le mot "idéologie", devenu un terme "passe-partout" d'une imprécision remarqualbe.) Pourtant, quelle que soit la perspective envisagée, l'étude du héros de roman ferait bien, nous semble-t-il, de se prévaloir des procédés de hiérarchisation que nous avons pratiqués ici, et qui s'avéreraient sans doute d'une utilité fondamentale à n'importe quel niveau d'analyse.

1 Northrop Frye, Anatomy of Criticism: Four Essays. (Princeton: Princeton University Press, 1956), 33-34.

2 B. V. Tomachevsky, "Thématique," Théorie de la littérature, ed. Tszvetan Todorov (Paris: Editions du Seuil, 1965), 295.

3 Philippe Hamon, Texte et idéologie. (Paris: PUF, 1984), 47.

4 Gerald Prince, "Introduction à l'étude du narrataire," Poétique 14, (1973), 188.

5 Gérard Genette, Figures III, (Paris: Editions du Seuil, 1972), 230.

6 Henri Mitterand souligne l'importance des traits physiques de certains personnages dans "Une anthropologie mythique: le système des personnages dans Thérèse Raquin et Germinal dans Le Discours du 
roman. (Paris: PUF, 1980), 49-67.

7 Tzvetan Todorov, Littérature et signification (Paris: Larousse, 1967), 70.

8 Dans son Essai sur les personnages des "Liaisons dangereuses" en tant que types littéraires. (Paris: Lettres Modernes, 1960), A.O. Aldridge affirme que: "Les critiques s'accordent pour voir dans la Marquise de Merteuil le personnage principal du roman." (p.5).

9 Laurent Versini, Le roman épistolaire, (Paris: PUF, 1979), 158.

10 Dans l'analyse de A.O. Aldrige citée ci-dessus, on trouve la constatation suivante: "Si le personnage principal des Liaisons dangereuses et bien la Marquise de Merteuil, c'est par contre Mme de Tourvel qui est à coup sûr l'héroïne" (C'est nous qui soulignons.), p. 22. 